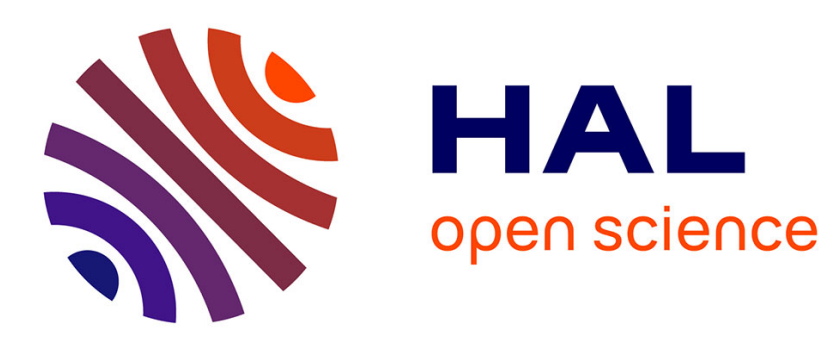

\title{
Decentralized Adaptive Partitioned Approximation Control of Robotic Manipulators
}

\author{
Hayder F N Al-Shuka, Rui Song
}

\section{To cite this version:}

Hayder F N Al-Shuka, Rui Song. Decentralized Adaptive Partitioned Approximation Control of Robotic Manipulators. ROMANSY 22 - Robot Design, Dynamics and Control, 2019, 10.1007/978-3319-78963-7_3. hal-01920186

\section{HAL Id: hal-01920186 https://hal.science/hal-01920186}

Submitted on 13 Nov 2018

HAL is a multi-disciplinary open access archive for the deposit and dissemination of scientific research documents, whether they are published or not. The documents may come from teaching and research institutions in France or abroad, or from public or private research centers.
L'archive ouverte pluridisciplinaire HAL, est destinée au dépôt et à la diffusion de documents scientifiques de niveau recherche, publiés ou non, émanant des établissements d'enseignement et de recherche français ou étrangers, des laboratoires publics ou privés. 


\title{
Decentralized Adaptive Partitioned Approximation Control of Robotic Manipulators
}

\author{
Hayder F. N. Al-Shuka and Rui Song \\ School of Control Science and Engineering, Shandong University, Jinan, China \\ hayder.al-shuka@rwth-aachen.de,rsong@sucro.org
}

\begin{abstract}
Partitioned approximation control is avoided in most decentralized control algorithms; however, it is essential to design feedforward control with improved tracking accuracy. As a result, this work is focused on decentralized adaptive partitioned approximation control for complex robotic systems using the orthogonal basis functions as strong approximators. In essence, the partitioned approximation technique is intrinsically decentralized with some modifications. The proposed decentralized control law consists of three terms: the partitioned approximation-based feedforward term that is necessary for precise tracking, the high gain-based feedback term, and the adaptive sliding gainbased term for compensation of modeling error. The passivity property is essential to prove the stability of local stability of the individual subsystem with guaranteed global stability. A two-link robot is simulated to verify the effectiveness of the proposed technique.
\end{abstract}

Keywords: Adaptive Approximation Control, Decentralized Control, Orthogonal Basis Functions.

\section{Introduction}

For complex robotic systems, such as humanoid robots or any robot having a number of degrees of freedom (DOFs) larger than 6-DOFs, difficulties are encountered in the implementation of the control algorithms. Therefore, over 30 years, the robotics researchers have focused on the problem of computational efficiency; see [1] for more details. However, the adaptive control algorithm that deals with controlling the robotic systems despite their uncertain parameters may decrease the computational efficiency of the dynamics $\mathrm{O}(n)$ algorithms [2]. Therefore, most researchers have used a local controller (decentralized controller) such as PID family for controlling complex dynamic systems [3]. However, Liu [4] has proved that decentralized PD control for robotic manipulator can ensure local stability without guaranteed global stability. Therefore, the author proposed decentralized a PD + nonlinear cubic controller with guaranteed global stability. Yang et al. [5] have used disturbance observer and adaptive sliding mode control for compensation of low-pass and high-pass coupled uncertainties respectively. In general, most available control strategies are based on designing PD control plus robust and or/adaptive term(s) for compensation of modeling 
errors and the interconnections. For more details on decentralized robust/adaptive control, see [5] and the references therein. Apart from conventional robust control, adaptive approximation control is a powerful tool to control robotic manipulators with unknown dynamics. There are two possibilities of adaptive approximation techniques: lumped (global) approximation-based adaptive control and partitioned (local) approximation-based adaptive control [6]-[8]. The former deals with collecting the uncertainty in one term and using the linear-in-the-parameters property to represent the uncertainty in terms of weighting and basis-function vectors. Then the controller is selected based on updating the weighting vector using Lyapunov stability. This strategy requires nominal (ideal) model for the unknown parameters. On the other hand, partitioned approximation adaptive control approximates each dynamic coefficient matrix of the equation of motion of the target robot separately using the linear-in-theparameters property. The control law is then designed based on updating the weighting-coefficient matrices of the corresponding basis-function matrices using Lyapunov stability. The interesting point is that the partitioned approximation does not require a nominal model for the dynamic coefficients easing the control task. However, most decentralized approximation control algorithms used lumped approximation term combined with either PD term or nominal values [9]-[11].

In view of above, partitioned approximation control is avoided in most decentralized control algorithms despite its importance in the design of feedforward control with improved tracking accuracy. As a result, this work is concerned with decentralized adaptive partitioned approximation control for complex robotic systems using the orthogonal basis functions as strong approximators. In essence, the partitioned approximation technique is intrinsically decentralized with some modifications. The proposed decentralized control law consists of three terms: the partitioned approximation-based feedforward term that is necessary for precise tracking, the high gain-based feedback term, and the adaptive sliding gain-based term for compensation of modeling error. The passivity property is essential to prove the stability of local stability of the individual subsystem with guaranteed global stability.

The remainder of the paper can be organized as follows. Methodology is presented in Section 2. Section 3 described simulation results and discussions. Section 4 concludes.

Remark 1. Preliminaries for adaptive approximation control and the related theorems such as Weierstrass theorem are described with some details in [8, 13].

\section{Methods}

\subsection{Dynamics of Contact-Free Motion Robots}

The equation of motion for $n$-DOF robotic manipulators in free space can be expressed as

$$
\boldsymbol{D}(\boldsymbol{q}) \ddot{\boldsymbol{q}}+\boldsymbol{C}(\boldsymbol{q}, \dot{\boldsymbol{q}})+\boldsymbol{g}(\boldsymbol{q})+\tau_{f}(\boldsymbol{q}, \dot{\boldsymbol{q}})=\boldsymbol{\tau}
$$


where $\boldsymbol{D} \in R^{n}$ is the inertia matrix of the links, $\boldsymbol{C} \in R^{n}$ is the Coriolis and centripetal matrix of the links, $g \in R^{n}$ is the gravity vector, $q \in R^{n}$ represents the angular joint displacement, $\tau_{f} \in R^{n}$ is the friction joint torque vector, and $\tau \in R^{n}$ denotes to the output joint torques.

Based on Eq. (1), the $k$ - th subsystem of the target robot, where $k \in n$ can be expressed as

$$
\sum_{j=1}^{n} d_{k j}(q) \ddot{q}_{j}+\sum_{j=1}^{n} c_{k j}(q, \dot{q}) \dot{q}_{j}+g_{k}(q)+\tau_{f k}\left(q_{k}, \dot{q}_{k}\right)=\tau_{k}
$$

The inertia, Coriolis and centripetal, and the gravity terms of Eq. (2) include coupled nonlinear functions, therefore, they are written in terms of general state variables.

The objective of decentralized control is to control every DOF individually; therefore, Eq. (2) can be further expressed as

$$
d_{k k}(q) \ddot{q}_{k}+c_{k k}(q, \dot{q}) \dot{q}_{k}+\Delta_{k}(q, \dot{q})=\tau_{k}
$$

with

$$
\Delta_{k}=\sum_{\substack{j=1 \\ j \neq k}}^{n} d_{k j}(q) \ddot{q}_{j}+\sum_{\substack{j=1 \\ j \neq k}}^{n} c_{k j}(q, \dot{q}) \dot{q}_{j}+g_{k}(q)+\tau_{f k}\left(q_{k}, \dot{q}_{k}\right)
$$

The following properties assumptions are necessary for adaptive approximation control assuming robotic manipulators with revolute joints $[3,12]$.

Property 1. The inertia matrix, Coriolis and centrifugal matrix, and the gravity vectors are uniformly bounded.

This property can be extended such that the dynamic coefficients and the disturbance term $\left(d_{k k}, d_{k j}, c_{k k}, c_{k j}, g_{k}, \tau_{f k}\right)$, with $k, j \in n$ are uniformly bounded.

Property 2. The matrix $\boldsymbol{M}=\dot{\boldsymbol{D}}-2 \boldsymbol{C}$ is a skew-symmetric matrix, i.e., $\boldsymbol{s}^{T} \boldsymbol{M} \boldsymbol{s}=0, \forall \boldsymbol{s} \in R^{n}$, if $\boldsymbol{C}(\boldsymbol{q}, \dot{\boldsymbol{q}})$ is defined using the Christoffel symbols.

Accordingly, the diagonal elements of $\boldsymbol{M}$ are equal to zero, i.e., $m_{i i}=0, \forall i \in n$.

The last property is important in deriving the stability of the proposed controller.

Assumption 1. The state variables of the target robots are measureable and bounded.

Assumption 2. Each entry of dynamic matrices of $\boldsymbol{D}, \boldsymbol{C}, \boldsymbol{g}$, and $\boldsymbol{\tau}_{f}$ satisfies conditions of Weierstrass approximation theorem [13], i.e., they can be represented as a linear combination of basis functions. 


\subsection{Decomposition Technique}

Let us recall the partitioned approximation technique described in details in [8]. Accordingly, Eq. (1) can be expressed as

$$
\left[\begin{array}{ccc}
\boldsymbol{w}_{D 11}^{T} \boldsymbol{\varphi}_{D 11} & \cdots & \boldsymbol{w}_{D 1 n}^{T} \boldsymbol{\varphi}_{D 1 n} \\
\vdots & \ddots & \vdots \\
\boldsymbol{w}_{D n 1}^{T} \boldsymbol{\varphi}_{D n 1} & \cdots & \boldsymbol{w}_{D n n}^{T} \boldsymbol{\varphi}_{D n n}
\end{array}\right] \ddot{\boldsymbol{q}}+\left[\begin{array}{ccc}
\boldsymbol{w}_{C 11}^{T} \boldsymbol{\varphi}_{C 11} & \cdots & \boldsymbol{w}_{C 1 n}^{T} \boldsymbol{\varphi}_{C 1 n} \\
\vdots & \ddots & \vdots \\
\boldsymbol{w}_{C n 1}^{T} \boldsymbol{\varphi}_{C n 1} & \cdots & \boldsymbol{w}_{C n n}^{T} \boldsymbol{\varphi}_{C n n}
\end{array}\right] \dot{\boldsymbol{q}}+\left[\begin{array}{c}
\boldsymbol{w}_{\Delta l}^{T} \boldsymbol{\varphi}_{\Delta l} \\
\vdots \\
\boldsymbol{w}_{\Delta n}^{T} \boldsymbol{\varphi}_{\Delta n}
\end{array}\right]+\boldsymbol{\varepsilon}=\boldsymbol{\tau}
$$

where $\boldsymbol{w}_{(.)} \in R^{\beta}, \boldsymbol{\varphi} \in R^{\beta}$ represent the weighting-coefficient and basis-function vectors respectively, and $\varepsilon \in R^{n}$ denotes to the accumulated modeling error vector. According to Eq. (4), the partitioned approximation technique has inherently decentralization features that can be exploited for decentralized control. In view of properties 1 , 2, assumptions 1, 2, and Eq. (4), Eq. (3) can be reformulated as

$$
\boldsymbol{w}_{D k}^{T} \boldsymbol{\varphi}_{D k} \ddot{q}_{k}+\boldsymbol{w}_{C k}^{T} \boldsymbol{\varphi}_{C k} \dot{q}_{k}+\boldsymbol{w}_{\Delta k}^{T} \boldsymbol{\varphi}_{\Delta k}+\varepsilon_{k}=\tau_{k}
$$

where

$$
d_{k k}(q)=\boldsymbol{w}_{D k}^{T} \boldsymbol{\varphi}_{D k}+\varepsilon_{D k}, c_{k k}(q)=\boldsymbol{w}_{C k}^{T} \boldsymbol{\varphi}_{C k}+\varepsilon_{C k}, \Delta_{k}(q)=\boldsymbol{w}_{\Delta k}^{T} \varphi_{\Delta k}+\varepsilon_{\Delta k}
$$

with $\varepsilon_{(.)}$is the corresponding modeling error. Accordingly, the dynamics of the coupled robotic system is transformed to decoupled subsystems based on linear combinations of basis function with constant weighting coefficients that should be updated using the Lyapunov theory.

Remark 2. A significant property that is inherently featured by Eq. (5) is that the whole system can freely be transformed to arbitrary subsystems. For example, a 6 DOF robot can be decoupled to two 3-DOF robotic subsystems, and so on.

\subsection{Controller Design}

The intuitive control law can be selected as

$$
\tau_{k}=\hat{d}_{k k}(q) \dot{v}_{k}+\hat{c}_{k k}(\dot{q}) v_{k}+\hat{\Delta}_{k}-K_{k} s_{k}-\hat{\Gamma}_{k}(t) \operatorname{sgn}\left(s_{k}\right)
$$

with $\hat{d}_{k k}=\hat{\boldsymbol{w}}_{D k}^{T} \varphi_{D k}, \hat{c}_{k k}=\hat{\boldsymbol{w}}_{C k}^{T} \varphi_{C k}, \hat{\Delta}_{k}=\hat{\boldsymbol{w}}_{\Delta k}^{T} \varphi_{\Delta k}, v_{k}=\dot{q}_{d k}-\Lambda_{k} e_{k}$,

$$
s_{k}=\dot{q}_{k}-v_{k}=\dot{e}_{k}+\Lambda_{k} e_{k}, e_{k}=q_{k}-q_{d k}
$$

where the symbol $(\hat{*})$ refers to the estimation, $K_{k}$ and $\Lambda_{k}$ are positive feedback gains. $\hat{\Gamma}_{k}(t)$ denotes to the adaptive sliding gain that should be updated in order to 
avoid the demand of modeling error bounds, please for more details on adaptive sliding mode control see [14], and

$$
\operatorname{sgn}(s)=\frac{|s|}{s}
$$

The mathematical relationship of Eq. (8) is very useful in the stability proof discussed later. Substituting Eq. (7) into Eq. (3a) leads to the output position closed loop dynamics

$$
d_{k k} \dot{s}+c_{k k} s_{k}+K_{k} s_{k}+\hat{\Gamma}_{k}(t) \operatorname{sgn}\left(s_{k}\right)=-\left(\tilde{\boldsymbol{w}}_{D k}^{T} \boldsymbol{\varphi}_{D k} \dot{v}_{k}+\tilde{\boldsymbol{w}}_{C k}^{T} \boldsymbol{\varphi}_{C k} v_{k}+\tilde{\boldsymbol{w}}_{\Delta k}^{T} \boldsymbol{\varphi}_{\Delta k}\right)+\varepsilon_{k}
$$

From Eq. (9), the closed loop control system is stable if $\tilde{\boldsymbol{w}}_{(.)} \rightarrow 0, \varepsilon_{k} \rightarrow 0$, and $\hat{\Gamma}_{k}(t) \rightarrow \delta_{k} \geq\left|\varepsilon_{k}\right|$. This requires finding suitable update laws for the weighting coefficients and the adaptive sliding gain. Let us select the following updating adaptive laws for the weighting vectors and adaptive sliding gain as follows.

$$
\dot{\hat{\boldsymbol{w}}}_{D k}=-\boldsymbol{Q}_{D k} \boldsymbol{\varphi}_{D k} \dot{v}_{k} s_{k}, \dot{\hat{\boldsymbol{w}}}_{C k}=-\boldsymbol{Q}_{C k} \boldsymbol{\varphi}_{C k} v_{k} s_{k}, \dot{\hat{\boldsymbol{w}}}_{\Delta k}=-\boldsymbol{Q}_{\Delta k} \boldsymbol{\varphi}_{\Delta k} s_{k}, \dot{\hat{\Gamma}}_{k}(t)=\frac{\left|s_{k}\right|}{\rho_{k}}
$$

where $\boldsymbol{Q}_{(.)} \in R^{\beta \times \beta}$ is a positive-definite adaptation matrix, and $\rho_{k}$ is an adaptation gain.

Theorem 1. The $k$-th subsystem of the robotic manipulator, $k \in n$, described by Eq. (5) combined with the input control law of Eq. (7), and with the adaptation laws of the weighting vectors, and the adaptive sliding gain of Eq. (10) is stable in the sense of Lyapunov stability [15]-[17].

\section{Proof.}

Consider the following Lyapunov-like function $\left(V_{k}\right)$ along the trajectory of Eq. (9)

$$
V_{k}=\frac{1}{2} d_{k k} s_{s}^{2}+\frac{1}{2} \tilde{\boldsymbol{w}}_{D k}^{T} \boldsymbol{Q}_{D k}^{-1} \tilde{\boldsymbol{w}}_{D k}^{T}+\frac{1}{2} \tilde{\boldsymbol{w}}_{C k}^{T} \boldsymbol{Q}_{C k}^{-1} \tilde{\boldsymbol{w}}_{C k}^{T}+\frac{1}{2} \tilde{\boldsymbol{w}}_{\Delta k}^{T} \boldsymbol{Q}_{\Delta k}^{-1} \tilde{\boldsymbol{w}}_{\Delta k}^{T}+\frac{1}{2} \rho_{k} \tilde{\Gamma}_{k}^{2}
$$

Taking the derivative of the last equation leads to

$$
\dot{V}_{k}=d_{k k} s_{k} \dot{\boldsymbol{s}}_{k}+\frac{1}{2} \dot{d}_{k k} s_{k}^{2}-\tilde{\boldsymbol{w}}_{D k}^{T} \boldsymbol{Q}_{D k}^{-1} \dot{\hat{\boldsymbol{w}}}_{D k}-\tilde{\boldsymbol{w}}_{C k}^{T} \boldsymbol{Q}_{C k}^{-1} \dot{\hat{\boldsymbol{w}}}_{C k}-\tilde{\boldsymbol{w}}_{\Delta k}^{T} \boldsymbol{Q}_{\Delta k}^{-1} \dot{\hat{\boldsymbol{w}}}_{\Delta k}-\rho_{k} \tilde{\Gamma}_{k}(t) \dot{\hat{\Gamma}}_{k}(t)
$$

By substituting Eq. (9) into Eq. (12), using the adaptation laws of Eq. (10), and applying passivity property 2 lead to

$$
\dot{V}_{k}=-K_{k} s_{k}^{2}+s_{k} \varepsilon_{k}-s_{k} \hat{\Gamma}_{k}(t) \operatorname{sgn}\left(s_{k}\right)-\left(\delta_{k}-\hat{\Gamma}_{k}(t)\left|s_{k}\right|\right.
$$


Using Eq. (8) yields

$$
\dot{V}_{k}=-K_{k} s_{k}^{2}+s_{k} \varepsilon_{k}-\delta_{k}\left|s_{k}\right|<0
$$

Both $s_{k}$ and $\tilde{\Gamma}_{k}(t)$ reach zero in finite time resulting in position error approaching to zero provided that $\delta_{k}<\left|\varepsilon_{k}\right|$.

Remark 3. Although $\delta_{k}$ should be larger than or equal to the upper bound of the modeling error, it is not included in the design of the proposed control law. Accordingly, the proposed control law of Eq. (7) does not require the bounds of the modeling error.

\section{Simulation Results and Discussions}

A simple 2-link manipulator is simulated to prove the validity of the proposed controller (Fig. 1). The parameters of the simulated manipulators are borrowed from [17]. It is assumed that the two joints are actuated with direct drive (gear ratio=1). The target manipulator moves freely (without constrained motion) with the following desired trajectories [16]: $q_{d 1}=30^{\circ}(1-\cos (2 \pi t))$ and $q_{d 2}=45^{\circ}(1-\cos (2 \pi t))$.

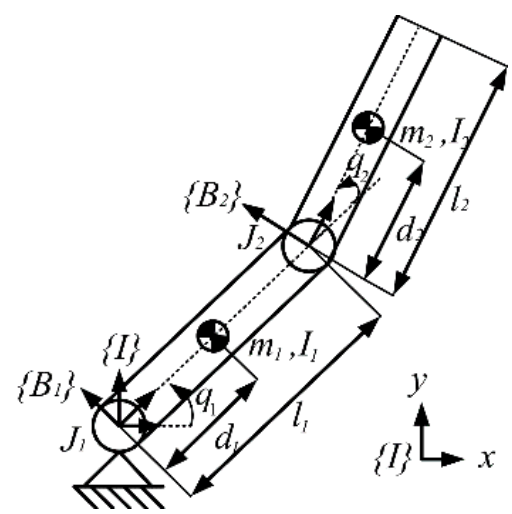

Fig. 1. The 2-link manipulator

Accordingly, the control law of Eq. (7) with the corresponding updating adaptive laws of the weighting vectors, Eq. (10), is used for precise tracking the desired references. A decentralized control is applied to each link subsystem using the proposed algorithms. The number of terms of basis orthogonal basis functions is 15 neglecting the modeling error (assuming a sufficient number of basis functions are used). The feedback and the adaptation gains are as follows: $K_{\boldsymbol{k}}=100, \Lambda_{k}=75, \boldsymbol{Q}_{D k}=\boldsymbol{Q}_{C k}=\boldsymbol{I}_{15}, \boldsymbol{Q}_{\Delta k}=0.01 \boldsymbol{I}_{15}$.

The adaptive partitioned approximation control (APAC) is compared with the decoupled proportional-derivative controller (PD) that is used extensively for control of 
robotic manipulators. The PD control law can be described as (Liu, 1999 [4]): $\tau_{k}=-K_{k} s_{k}=-K_{k}\left(\dot{e}_{k}+e_{k}\right)$, with $K_{k}=100$. Figures 2 and 3 show the position errors and the input control torques respectively considering both the APAC and PD controllers. Both controllers work well with small position error; however, the APAC shows more accuracy than the PD considering the same high gain. One important point should be mentioned is that although the APAC control law does not track precisely the dynamic coefficients of the equation of motion for each subsystem, the controller tracks well the desired references. The system signals should be persistently excited in order to ensure good estimation for unknown parameters [13].
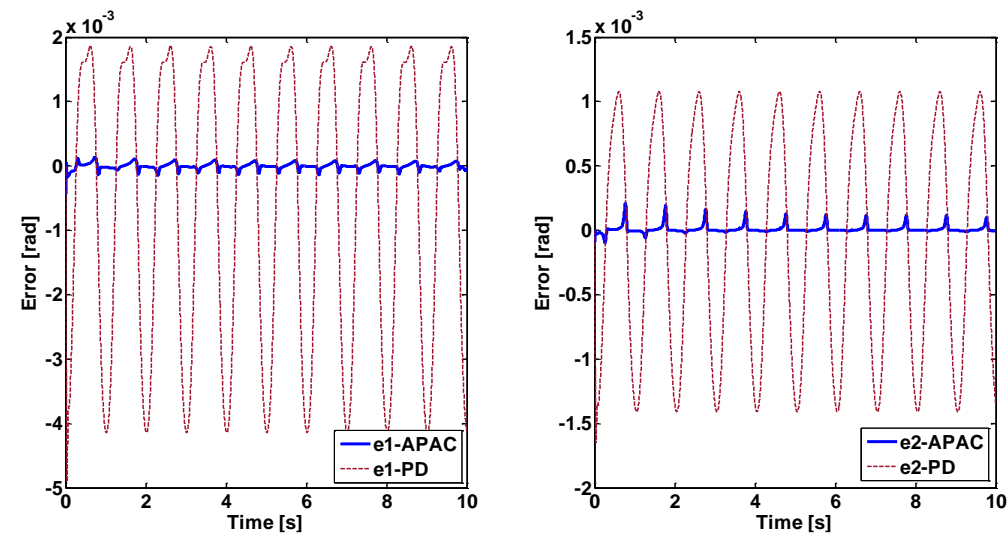

Fig. 2. The position error for the 2-link robot
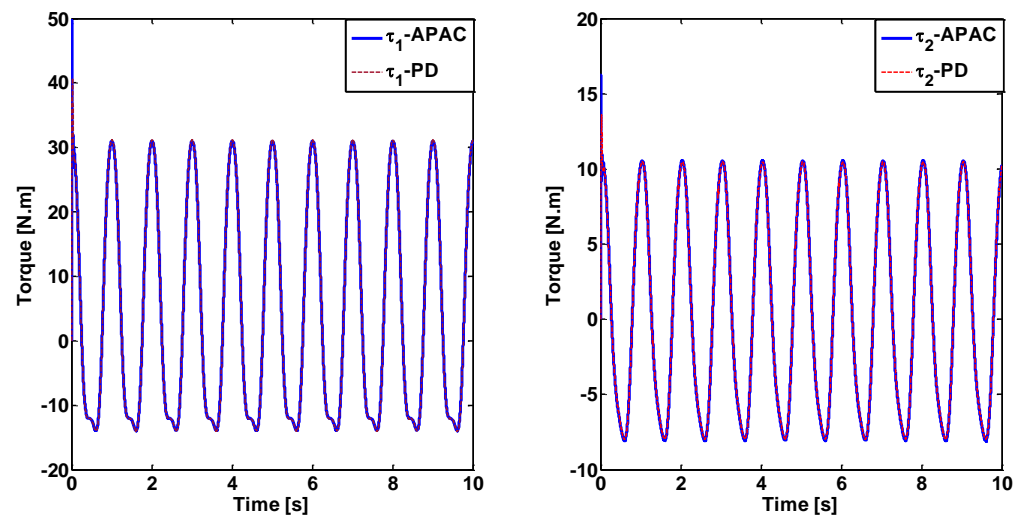

Fig. 3. The input control torques for the 2-link robot.

\section{Conclusions}

This paper proposes decentralized adaptive control based on partitioned approximation technique. The control law consists of three terms: adaptive partitioned approxi- 
mation-based feedforward term, high-gain feedback term, and an adaptive sliding term for compensation for modeling error. Further work is required to consider the joint flexibility and actuator dynamics.

\section{References}

1. Saha, S. K.: Recursive dynamics algorithms for serial, parallel and closed-chain multibody systems. In: Indo-US Workshop on Protein Kinematics and Protein Conformations, IISC, 1-34. Bangalore (2007).

2. Fu, K. S., Gonzalez, R. C., Lee C. S. G.: Robotics: control, sensing, vision, and intelligence. McGraw-Hill Book Company, USA (1987).

3. Spong, M. W., Vidyasagar, M.: Robot dynamics and control. John Wiley \& Sons, Inc. USA (1989).

4. Liu, M.: Decentralized control of robot manipulators: nonlinear and adaptive approaches. IEEE Transactions on Automatic Control 44, 357-363 (1999).

5. Yang, Z.-J., Fukushima, Y., Qin, P.: Decentralized adaptive robust control of robot manipulators using disturbance observers. IEEE Transactions on Control Systems Technology 20, 1357-1365 (2012).

6. Lewis, F. L., Yesildirek, A., Liu, K.: Neural net robot controller: structure and stability proofs. J. Intell. Robot. Syst. 13, 1-23 (1995).

7. Lewis, F. L., Yesildirek, A., Liu, K.: Multilayer neural net robot controller with guaranteed tracking performance. IEEE Trans. Neural Netw.7, 1-12 (1996).

8. Al-Shuka, H. F. N.: On local approximation-based adaptive control with applications to robotic manipulators and biped robots. Int. J. Dynam. Control 6 (1), 339-353 (2018).

9. Panagi, P., Polycarpou, M.. Decentralized Adaptive approximation based control of a class of large-scale systems, In: American Control Conference, 92-97. IEEE, Washington, USA, (2008).

10. Tan, K., Huang, S., Lee, T. H.: Decentralized adaptive controller design of large-scale uncertain robotic systems. Automatica 45:161-166 (2009).

11. Fateh, M., Fateh, S.: Decentralized direct adaptive fuzzy control of robots using voltage control strategy. Nonlinear Dyn 70, 1919-1930 (2012).

12. Mulero-Martínez, J. I.: Uniform bounds of the coriolis/centripetal matrix of serial robot manipulators. IEEE Transactions on Robotics 23, 1083 - 1089 (2007).

13. Farrell, J., Polycarpou, M.: Adaptive approximation based control: unifying neural, fuzzy and traditional adaptive approximation approaches. John Wiley \& Sons, Inc., USA (2006).

14. Utkin, V. I., Poznyak, A. S.: Adaptive sliding mode control. In: Bandyopadhyay B., Janardhanan S., Spurgeon, S. (eds.) Advances in Sliding Mode Control. Lecture Notes in Control and Information Sciences, vol. 440, pp. 21-53. Springer, Berlin, Heidelberg (2013).

15. Al-Shuka, H. F. N.: Dynamic modeling, walking pattern generators and adaptive control of biped robot. PhD Dissertation. RWTH Aachen University, Germany (2014).

16. Slotine, J. -J. E., Li, W.: Applied nonlinear control. Prentice-Hall, Inc., USA (1991).

17. Huang, A. -C., Chien, M. -C.: Adaptive control of robot manipulators: a unified regressor free approach. World Scientific Publishing Co. Pte. Ltd. Singapore (2010). 